Palavras chave: sensaios não destrutivos ultrassom inspeção

Histórico: Recebido 22/01/2014 Aceito 10/09/2015

Keyworks: nondestructive test ultrasound inspection

Correspondence: lourencopp@gmail.com
Lourenço Panosso Perlin', Marcela Alejandra Juliani', Ângela do Valle', Roberto Caldas de Andrade Pinto'

\section{FUNDAMENTAÇÃO MATEMÁTICA DA TOMOGRAFIA ULTRASSÔNICA NA AVALIAÇÃO DA INTEGRALIDADE DE ELEMENTOS ESTRUTURAIS EM MADEIRA}

RESUMO: A tomografia ultrassônica é uma técnica não destrutiva com grande potencial na avaliação da integridade de seções transversais de elementos de madeira. São várias as pesquisas que abordam a influência de diversos fatores como umidade, presença de fungos, entre outros na obtenção de tomogramas a partir da utilização de softwares e equipamentos comerciais projetados para este fim. A fundamentação matemática desta técnica, por outro lado, quando abordada, ocorre de uma forma simplificada. $O$ objetivo desse trabalho é apresentar o processamento matemático envolvido na técnica de tomografia ultrassônica e assim permitir o desenvolvimento de uma ferramenta computacional própria. Resultados preliminares de detecção de imperfeições internas em uma peça de madeira a partir de uma ferramenta computacional, inicialmente desenvolvida, são apresentados e discutidos. Os resultados obtidos identificam a presença de imperfeições internas, e exibem a influência que a anisotropia da madeira exerce sobre a propagação dos pulsos ultrassônicos.

\section{MATHEMATICAL BASIS OF ULTRASONIC TOMOGRAPHY FOR INTEGRITY EVALUATION IN WOOD STRUCTURAL ELEMENTS}

ABSTRACT: Ultrasonic tomography is a non-destructive technique used in the internal evaluation of wooden elements. Usually, research in this area lies in the influence of different factors in the obtained tomograms given by commercial software. The mathematical fundamentals of tomography are sparsely discussed. The aim of this study is to present the mathematical process, and thus to allow the implementing of a specific computational tool. This paper presents preliminary results for detecting internal flaws in wood specimens. The generated images identify the presence of the flaws, and show the influence of the material anisotropy in the ultrasonic pulse velocity. 


\section{INTRODUÇÃO}

Constantes agressões de origem ambiental são uma realidade para todas as construções. Tratando especificamente das edificações cujos elementos estruturais são feitos em madeira, as variações ambientais de umidade e temperatura, a presença de trincas, fungos, insetos, entre outros fatores, modificam a estrutura da mesma, provocando perda de massa do material, o que reflete diretamente no módulo de elasticidade e na sua resistência mecânica.

Técnicas não destrutivas de propagação de ondas de tensão podem ser utilizadas na avaliação destas peças. A velocidade de propagação de um pulso ultrassônico depende das propriedades elásticas e densidade do meio em que se propaga (BRANCHERIAU e BAILLERES, 2002). A tomografia baseada na velocidade de propagação da onda ultrassônica vem sendo utilizada na detecção de não homogeneidades em seções transversais de elementos de madeira (PRIETO e LASAYGUES, 20l4). Muitos são os trabalhos desenvolvidos com o emprego de softwares comerciais como: ARBOTOM $®$ (AMODEl et al., 20l0; LATORRACA et al., $20 \mathrm{I}$ I), FAKOPP ${ }^{\circledR}$ (CANDIAN e SALES, 2009; PADUELLI, 20II), MIGRATON® (MARTINIS et al., 2004; SAMBUELLI et al., 2003) e PICUS ${ }^{\circledR}$ (DEFLORIO et al., 2007; WANG et al., 2009).

No Brasil, há um grupo desenvolvendo um método tomográfico próprio, baseado na média de faixas de leituras executadas sobre uma malha em torno do objeto ensaiado (GONÇALVES et al., 201 I ; SECCO et al., 20।2).

Objetivo de todos estes trabalhos supracitados é a avaliação de diversos fatores, tais como a frequência dos transdutores, a umidade do corpo de prova, o perfil radial do lenho, a presença de vazios internos, no resultado tomográfico. Entretanto, os procedimentos matemáticos envolvidos no processamento tomográfico, quando abordados, ocorreram de forma superficial.

O objetivo desse artigo é preencher essa lacuna, expondo o desenvolvimento matemático da tomografia ultrassônica, possibilitando o desenvolvimento de uma ferramenta computacional própria para uso de tomografia ultrassônica em madeira. Os tomogramas gerados pela ferramenta computacional em desenvolvimento são comparados às seções reais, íntegras e com vazios, testadas.

\section{MATERIAL E MÉTODOS}

\section{Fundamentação da Tomografia Ultrassônica}

A fundamentação matemática da tomografia, desenvolvida no início do século $X X$ (RADON, 1917) ficou conhecida como Transformada de Radon (DEANS, 1983). Sua aplicação, usando ondas mecânicas como medida física, resultou na tomografia ultrassônica, muito aplicada na área geotécnica (JACKSON e TWEETON, 1994) e na área dos ensaios não destrutivos.

Considera-se, inicialmente, que o pulso ultrassônico trafega em linha reta em um material isotrópico. Em uma leitura ultrassônica, como apresentado na Figura $\mathrm{I}-\mathrm{a}$, obtêm-se o tempo $\mathrm{T}$ de propagação entre dois transdutores. Como a distância $L$ entre os transdutores é conhecida, a velocidade do pulso (V) pode ser calculada pela Equação I,

$\mathrm{V}=\frac{\mathrm{L}}{\mathrm{T}} \quad \therefore \mathrm{T}=\frac{\mathrm{L}}{\mathrm{V}}$

$[1]$

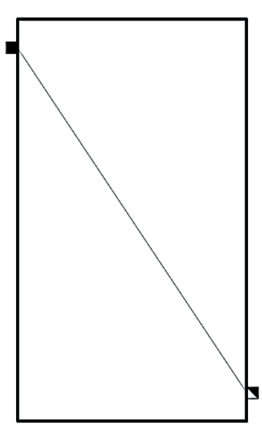

(a)

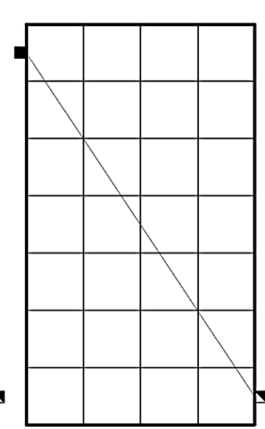

(b)

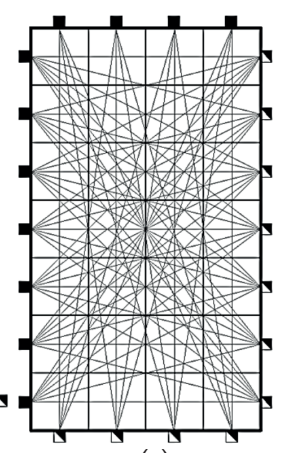

(c)
Fonte: Perlin e Pinto (2013)

FIGURA 1 Representação de leituras ultrassônicas - (a) em uma seção transversal - (b) em uma seção transversal discretizada em elementos - (c) várias leituras.

FIGURE 1 Ultrasonic reading representation - (a) in a cross section - (b) in a cross section divided into elements $-(c)$ several readings.

Ao discretizar a mesma a seção transversal em diferentes elementos, Figura I-b, o pulso ultrassônico trafegará em diferentes distâncias em cada elemento ao longo do caminho percorrido. Deste modo, o tempo total de propagação é obtido a partir dos tempos trafegados em cada elemento, como expresso pela Equação 2 (JACKSON; TWEETON, 1994), sendo $p_{j}$ é a vagarosidade de propagação no elemento $j$ (inverso da velocidade) e $d L_{j}$ a distância percorrida no elemento $j$. 
$\mathrm{T}=\sum_{\mathrm{j}=1}^{\mathrm{n}} \mathrm{p}_{\mathrm{j}} * \mathrm{dL}_{\mathrm{j}}$

Para possibilitar a utilização do processo tomográfico, são necessárias várias leituras ao longo da seção transversal, conforme exibe a Figura I-c. Desta forma, considerando $\mathrm{m}$ leituras, pode-se reescrever a Equação 2 na forma da Equação 3 (PERLIN e PINTO, 20I3), sendo $m$ é número total de leituras realizadas; $n$ o número total de elementos discretizados; $\underline{D}$ a matriz com $m$ linhas e $n$ colunas que armazena as distâncias percorridas pelas ondas ultrassônicas nos elementos $j$, quando realizado nas leituras $i$; $\underline{P}$ o vetor $\operatorname{com} n$ linhas que armazena as vagarosidades dos diferentes elementos discretizados $j$; e $I$ o vetor com $m$ linhas que armazena os tempos de todas as leituras $i$.

$$
\underline{T_{m}}=\underline{D_{m, n}} \cdot \underline{P_{n}}
$$

Como a malha e a posição dos transdutores são conhecidas para as diferentes leituras, a matriz $\underline{D}$ é determinada por simples geometria. Como os valores do vetor I são dados pelas leituras ultrassônicas, resta a determinação dos valores do vetor $\underline{P}$.

Dentre os diversos métodos de resolução de um sistema tomográfico, o processo iterativo de Cimmino, na forma otimizada por Jackson e Tweeton (1994), possibilita uma convergência mais rápida (PERLIN, 20I I). Denominado de Cimmino otimizado, também conhecido como SIRT - Simultaneous Iterative Reconstruction Technique, tal processo iterativo encontra-se na Equação 4 (PERLIN e PINTO, 20I3), sendo: $k$ é o número da iteração atual; $m$ o número de equações do sistema; $n$ o número de incógnitas do sistema; $\underline{P}_{n}{ }^{(k)}$ armazena os valores do passo iterativo atual $k ; \underline{P}^{(k-1)}$ armazena os valores do passo iterativo anterior $k-1$; e $\underline{W}_{\mathrm{m}, \mathrm{n}}$ a matriz construída pela Equação 5 , em que com $N_{j}$ sendo o número de equações, onde o termo da dimensão $j$ é diferente de zero, e $d_{i j}$ o termo da linha $i$ e da coluna $j$ da matriz $\underline{D}_{m, n}$.

$\underline{\mathrm{P}}^{(\mathrm{k})}=\underline{\mathrm{P}}^{(\mathrm{k}-1)}+\underline{\mathrm{W}, \mathrm{n}}^{\mathrm{T}} *\left[\underline{\mathrm{T}_{\mathrm{m}}}-\underline{\mathrm{D}_{\mathrm{m}, \mathrm{n}}} * \underline{\mathrm{P}}^{(\mathrm{k}-1)}\right]$

$\mathrm{w}_{\mathrm{ij}}=\frac{\mathrm{d}_{\mathrm{ij}}}{\mathrm{N}_{\mathrm{j}} * \sum_{\mathrm{k}=1}^{\mathrm{n}}\left(\mathrm{d}_{\mathrm{ik}}\right)^{2}}$

Obtidas as vagarosidades de cada elemento discretizado, as correspondentes velocidades são calculadas pela sua inversa e atribuídas ao ponto central do respectivo elemento. Para efetuar o cálculo das velocidades dos demais pontos do tomograma, utiliza-se a Equação 6, sendo: $\mathrm{V}_{h}^{(d)}$ é o vetor das velocidades dos $h$ pontos com velocidades desconhecidas; $\underline{V}_{n}$ é o vetor das velocidades dos $n$ pontos com velocidades conhecidas; $\zeta_{\mathrm{h}, \mathrm{n}}$ é a matriz dos pesos que cada ponto conhecido possui sobre o cálculo de cada ponto desconhecido. Para obter $\zeta_{i, j}$, deve-se utilizar alguma função interpoladora $f\left(d_{i, j}\right)$ em função da distância $d_{i, j}$. Neste trabalho, optou-se por uma função spline de terceiro grau para a interpolação necessária entre os valores de velocidade obtidos, conforme apresentado por Ruggiero e Lopes (1996).

$\underline{\mathrm{V}_{\mathrm{h}}^{(d)}}=\underline{\zeta_{\mathrm{h}, \mathrm{n}}}{ }^{*} \underline{\mathrm{V}}$

Um software de tomografia ultrassônica foi desenvolvido a partir da fundamentação matemática apresentada anteriormente. Este software, denominado TUCOM (Tomografia Ultrassônica em Concreto e Madeira), resolve o problema tomográfico, emitindo tomogramas de uma seção transversal a ser analisada.

\section{Programa Experimental}

De forma a validar o software em desenvolvimento, corpos de prova em madeira, com diferentes tamanhos de não homogeneidades internas, foram preparados em laboratório. Para tanto, um disco de tora de Pinus (Pinus elliottii) autoclavado com CCA tipo C (solução de Cromo, Cobre e Arsênio), com espessura de $9,6 \mathrm{~cm}$ e diâmetro aproximado de $37,0 \mathrm{~cm}$ (Figura 2-a) foi exposto a 12 ciclos de secagem em estufa a $50^{\circ} \mathrm{C}$, por períodos não superiores a I hora, de forma a reduzir a probabilidade de ocorrências de trincas. Um corpo de prova quadrado com aresta de $20 \mathrm{~cm}$ foi retirado do centro do disco seco (Figura 2-b). O material excedente foi aproveitado na confecção de corpos de prova de dimensões de $3 \times 4 \times 5 \mathrm{~cm}$, fornecendo um valor de densidade úmida média de $454 \mathrm{~kg} \cdot \mathrm{m}^{-3}$. Após secagem conforme NBR 7I90 (ABNT, 1997) obteve-se uma umidade média de $22,8 \%$.

\section{Ensaios Ultrassônicos}

Utilizou-se uma malha discretizadora de 2,5 $\mathrm{cm}$, com os pontos de leituras localizados exatamente no meio das faces externas dos elementos superficiais, 
conforme a Figura 3-a. Foram executadas leituras com todas as combinações possíveis de posição dos transdutores entre faces opostas (Figura 3-b), utilizando-se um transdutor exponencial de $30 \mathrm{kHz}$.

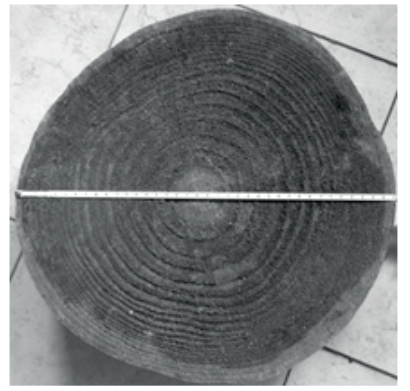

(a)

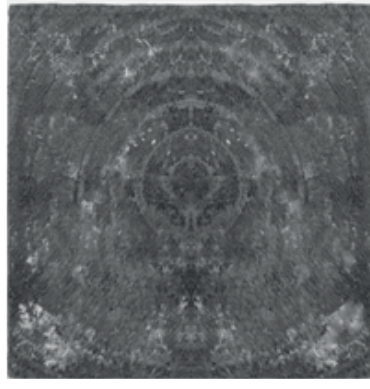

(b)
FIGURA 2 Pinus autoclavado - (a) disco original - (b) quadrado de $20 \mathrm{~cm}$.

FIGURE 2 Autoclaved pine- (a) original disk - (b) $20 \mathrm{~cm}$ square.

\section{Estados de Integridade Analisados}

Para se analisar a capacidade da tomografia ultrassônica, com a formulação apresentada, na detecção de vazios, 3 diferentes estados de integridade do corpo de prova foram analisados, conforme apresentados na Figura 4. Enquanto no primeiro estado (I), a peça encontrava-se íntegra, para o segundo estado (II) foi executada uma perfuração quadrada paralela às fibras, retirando completamente I elemento discretizado $(2,5 \times 2,5 \mathrm{~cm})$, Para o terceiro estado (III), o corpo de prova foi perfu-

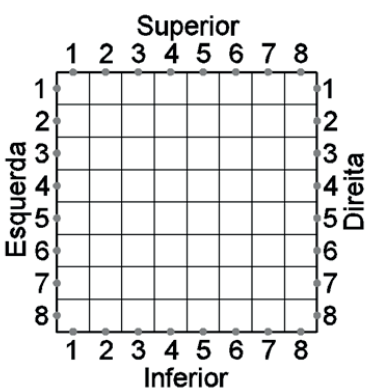

(a)

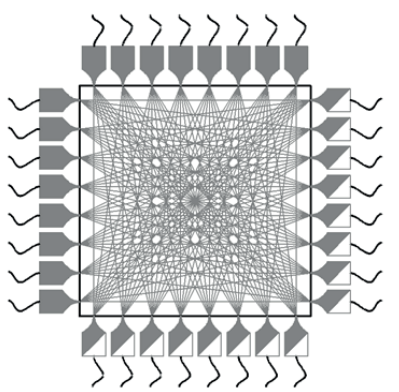

(b)
FIGURA 3 Malha adotada (a) pontos de leituras - (b) leituras executadas.

FIGURE 3 Adopted mesh (a) reading points - (b) performed reads.

rado em 4 elementos discretizados $(5,0 \times 5,0 \mathrm{~cm})$, novamente na direção paralela às fibras.

Para cada um dos 3 estados analisados, foram executadas as leituras ultrassônicas descritas no item 2.3. Os tempos de trânsito registrados dos pulsos ultrassônicos, a geometria do corpo de prova e a posição da malha discretizadora adotada foram inseridos no TUCOM (Tomografia Ultrassônica em Concreto e Madeira).

\section{RESULTADOS E DISCUSSÃO}

Os tomogramas obtidos para os estados de integridade analisados estão apresentados na Figura 5.

O tomograma do primeiro estado (Figura 5-a) apresentou uma seção relativamente homogênea, onde

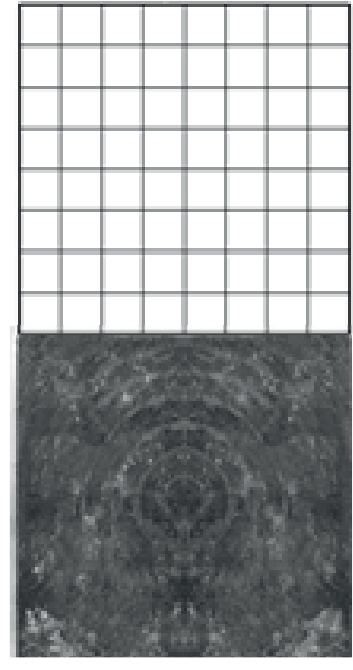

I

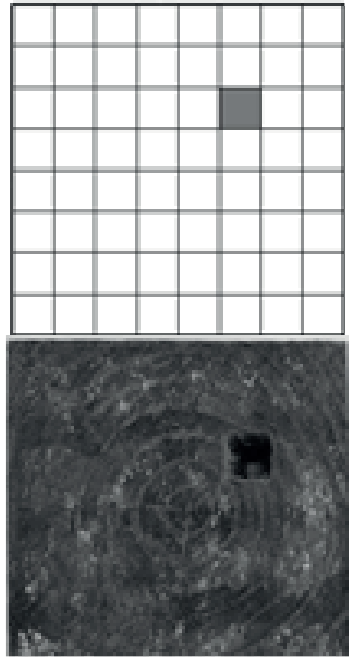

II

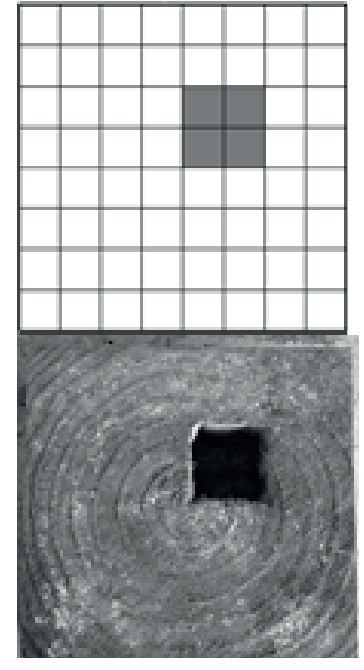

III

FIGURA 4 Corpos de prova testados.

FIGURE 4 Tested specimens. 


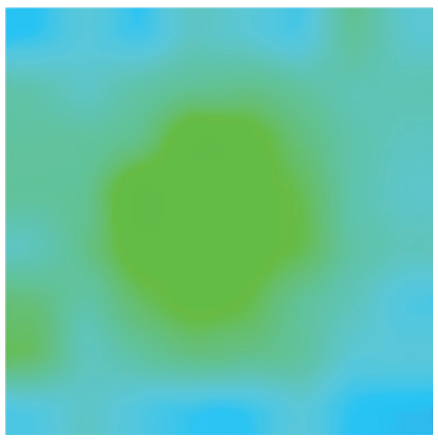

(a)

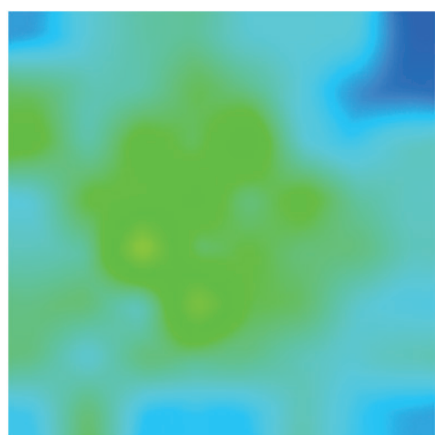

(b)

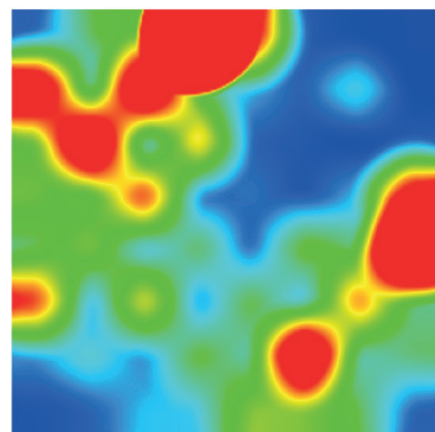

(c)

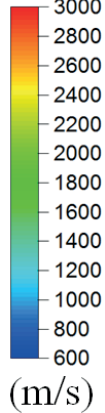

(d)

FIGURA 5 Tomogramas (a) Estado I (b) Estado II (c) Estado III (d) velocidades ( $\left.\mathrm{m} \cdot \mathrm{s}^{-1}\right)$.

FIGURE 5 Tomograms (a) Condition I (b) Condition II (c) Condition III (d) velocities $\left(\mathrm{m} \cdot \mathrm{s}^{-1}\right)$.

é possível notar que a região central obteve uma velocidade superior à da periferia. Analisando as 128 leituras efetuadas para este estado, suas respectivas velocidades médias e o afastamento c entre o trajeto retilíneo e a medula (Figura 6-a), verifica-se que, quanto maior a distância c, menor é a velocidade média do pulso ultrassônico (Figura 6-b). Este fato decorre da anisotropia do material, onde o trajeto de leitura próximo da direção radial (menores valores de c) possui velocidade superior à de um trajeto posicionado próximo à direção tangencial (maiores valores de $\mathrm{c}$ ).

A Figura 7-a evidencia que na região central predominavam leituras com trajeto próximo à direção radial. Sendo assim, como esperado, o processamento tomográfico conferiu a essa região velocidades maiores. Por outro lado, na periferia do corpo de prova (Figura 7-b), leituras de trajetos próximos das direções tangenciais predominavam, conferindo a essa área velocidades menores do que nas direções de propagação radial (BUCUR, 2006).

O tomograma do segundo estado (Figura 5-b)

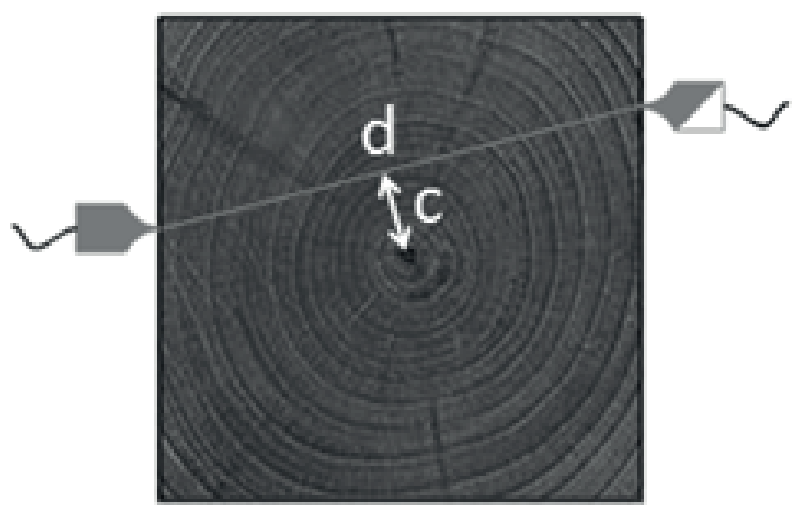

FIGURA 6 Análise das leituras do Estado I.

FIGURE 6 Readings analysis of Condition I. é resultado de um efeito conjunto da anisotropia da madeira e da presença da perfuração. É possível notar uma diminuição da velocidade do pulso ultrassônico no canto superior direito, demonstrando que a perfuração de 2,5 $x 2,5 \mathrm{~cm}$ influenciou no resultado da tomografia. Contudo, não é possível afirmar com base no resultado que naquela região existe um vazio.

No tomograma do terceiro estado (Figura 5-c), a presença da perfuração demonstrou-se muito mais influente que no segundo estado. Uma grande área de baixa velocidade encontra-se na parte superior direita do tomograma, demonstrando que as leituras foram afetadas pela perfuração e que há um indicativo da ocorrência de uma não homogeneidade nesta região.

Já com relação às zonas de alta velocidade do mesmo tomograma, estas aparecem como efeito da busca de equilíbrio do sistema tomográfico. Na Figura 8-a, estão exibidos os trajetos das leituras que sofreram variação relativa inferior a $10 \%$, quando comparadas as leituras entre os estados de integridade I e III.

Percebe-se que várias leituras que não foram

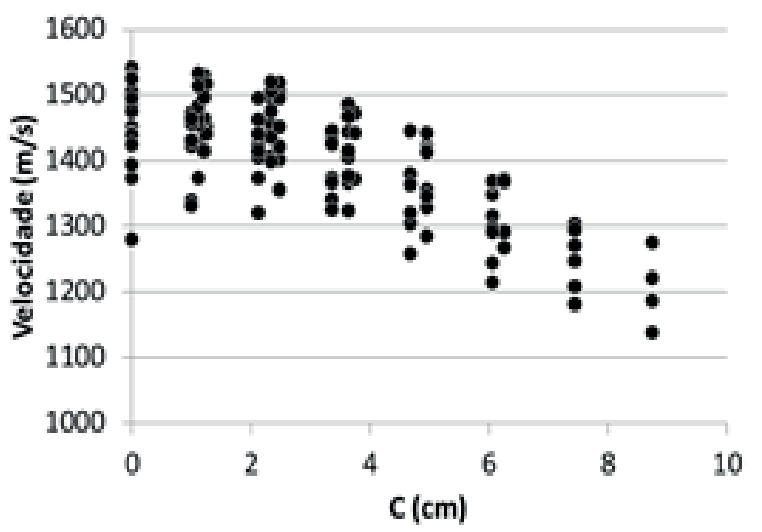




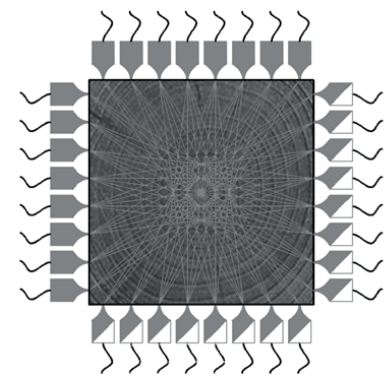

(a)

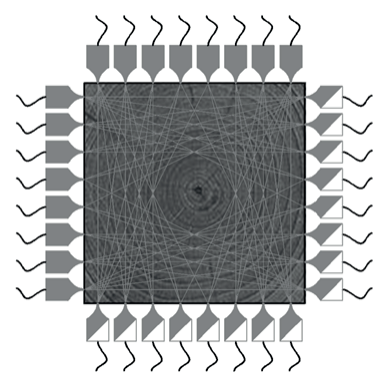

(b)
FIGURA 7 Leituras por regiões - (a) aprox. radiais - (b) aprox. tangenciais.

FIGURE 7 Readings by regions - (a) near radial - (b) near tangential. afetadas pela perfuração trafegam pela zona de baixa velocidade criada pelo processamento tomográfico. Por exemplo, a leitura Esquerda-I x Direita-I trafega pela região de baixa velocidade do lado direito do tomograma. Como esta leitura de tempo não sofreu variação significativa, o processamento tomográfico cria uma região de velocidade elevada na esquerda do tomograma, equilibrando e mantendo a leitura de tempo aproximadamente a mesma. $O$ mesmo efeito ocorre com várias outras leituras, entre elas: S4-15; S4-16; S4-17; S3-15; S3-16; S3-17; S3-18 (S Superior, I Inferior, E Esquerda, D Direita).

Por outro lado, na Figura 8-b estão apresentadas as leituras que sofreram variação relativa superior a $10 \%$, quando comparadas as leituras entre os estados de integridade I e III. Percebe-se que a grande maioria das leituras que sofreram alteração pela presença do furo foram as que utilizaram os pontos de leitura $\mathrm{S} 5, \mathrm{S6}, \mathrm{S7}$, S8, DI, D2, D3 e D4. Tais pontos de leituras são exatamente os que tinham trajeto coincidente com a di- reção radial e que foi seccionado pela perfuração (Figura 8-c). Este efeito justifica a maior alteração no tempo de propagação. $O$ valor de leitura do tempo para a posição S8-18, por exemplo, sofre variação de $24 \%$ entre os estados de integridade I e III, contudo, considerando sua trajetória retilínea, ela não passa pela área perfurada (Figura 8-b). Logicamente, quando o corpo de prova estava íntegro (Estado I), esta leitura possuía um tempo de trânsito menor e provavelmente seguia por caminhos radiais, que foram seccionados no Estado III.

Percebe-se, portanto, que as considerações iniciais aplicadas ao processamento tomográfico, que concebiam o pulso ultrassônico trafegando em linha reta em um material isotrópico, não permitem que o modelo reproduza as condições reais de peças de madeira. A ortotropia característica da madeira, sua natureza heterogênea, a presença de falhas e a orientação de grã, entre outros fatores, fazem com que o pulso ultrassônico, ao procurar trajetos de propagação mais rápidos, não necessariamente percorra caminhos retilíneos.

Por outro lado, a geração de imagens por tomografia ultrassônica, desenvolvida para materiais isotrópicos, foi capaz de auxiliar em um primeiro estudo na detecção de não homogeneidades internas em peças de madeira. Entretanto, a técnica requer a inserção das características anisotrópicas da madeira na formulação para que os tomogramas resultem mais precisos.

A comparação dos tomogramas obtidos com outras técnicas não destrutivas aos aqui apresentados poderia auxiliar ainda mais na validação da aplicação da técnica tomográfica na seção transversal de peças de madeira.

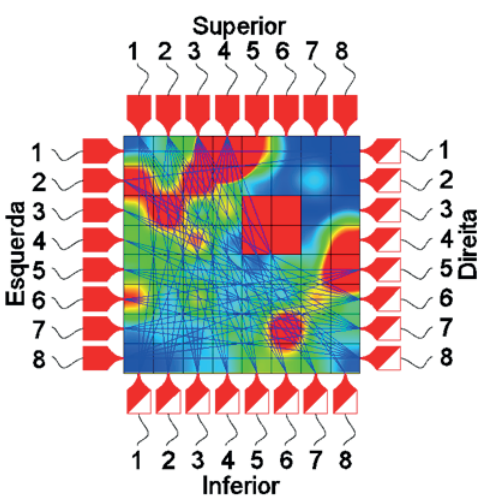

(a)

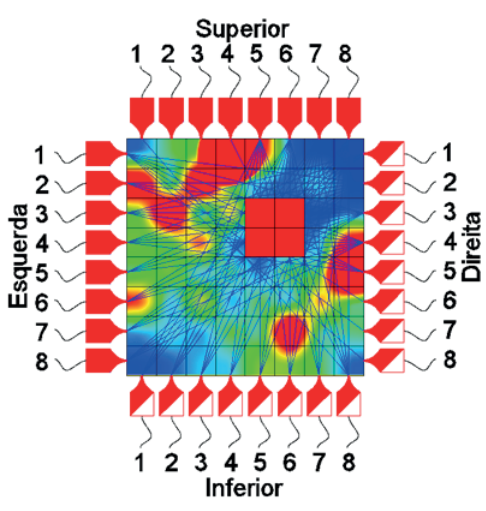

(b)

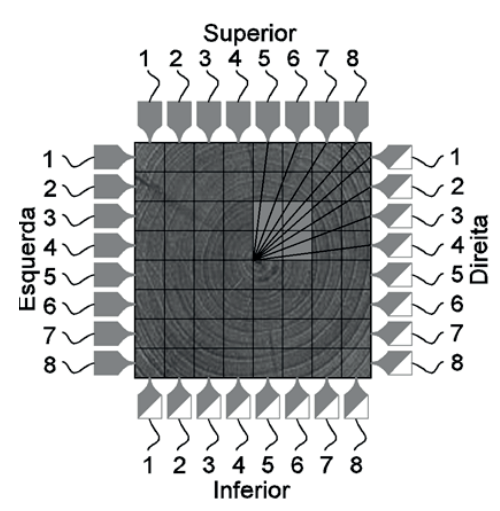

(c)

FIGURA 8 Leituras com variação relativa do Estado I para o Estado III (a) inferior a I0\% (b) igual e superior a I0\% (c) pontos de leituras cujo caminho na direção radial foi seccionado pela perfuração.

FIGURE 8 Readings that have suffered relative variation from Condition I to Condition III (a) below 10\% (b) equal and above I0\% (c) readings points whose radial direction was sectioned by drilling. 


\section{CONCLUSÕES}

Neste artigo demonstrou-se como é possível empregar os conceitos matemáticos da tomografia computadorizada, desenvolvidos por Radon, utilizando como medida física a velocidade do pulso ultrassônico. Comprovou-se que não se faz necessário o uso de equipamentos tomográficos específicos para utilizar esta técnica, que requer apenas um software tomográfico e um aparelho convencional de ultrassom para medir o tempo de trânsito do pulso ultrassônico. Os resultados apresentados demonstraram a potencialidade da técnica para analisar a seção transversal de peças de madeira. Diferentemente de outros materiais de construção, como - concreto e o aço, a madeira possui características próprias que impõem desafios maiores na aplicação da tomografia ultrassônica. Sua anisotropia e seu comportamento higroscópico devem ser levados em consideração quando da utilização do ultrassom para a localização de não homogeneidades internas no material.

\section{REFERÊNCIAS}

ABNT. NBR 7I90 - Projeto de estruturas de madeira. Rio de Janeiro: ABNT, 1997. 107p.

AMODEI, J. B.; OLIVEIRA, B. R. U.; GURGEL, M. D. M.; CARVALHO, A. M.; MEDEIROS, R. A.; LATORRACA, J. V. D. F. Avaliação preliminar da qualidade da madeira de Tectona grandis L. f. através da tomografia de impulso. Floresta e Ambiente, Rio de Janeiro, v. 17, n. 2, p. 124-128, 2010.

BRANCHERIAU, L.; BAILLERES, H. Natural vibration analysis of clear wooden beams: A theoretical review. Wood Science and Technology, New York, v. 36, p. 347-365, 2002.

BUCUR, V. Acoustics of Wood. New York: Springer, 2006. 393p.

CANDIAN, M.; SALES, A. Aplicação das técnicas não-destrutivas de ultra-som, vibração transversal e ondas de tensão para avaliação de madeira. Ambiente Construído, Porto Alegre, v. 9, n. 4, p. 83-98, out. 2009.

DEANS, S. R. The Radon Transform and some of its applications. New York: John Wiley \& Sons, 1983. 289p.

DEFLORIO, G.; FINK, S.; SCHWARZE, F. W. M. R. Detection of incipient decay in tree stems with sonic tomography after wounding and fungal inoculation. Wood Science and Technology, Munich, v. 42, n. 2, p. II7-132, I7 out. 2007.

GONÇALVES, R.; SECCO, C. B.; CERRI, D. G. P.; TRINCA, A. J.; LORENSANI, R. G. M. Tomografia ultrassônica para in- speção de toras e de árvores. Revista Abende, São Paulo, v. 46, p. $40-43,201$ I.

JACKSON, M. J.; TWEETON, D. R. MIGRATOM - Geophysical tomography using wavefront migration and fuzzy constraints. Washington: U.S. Department of Interior Bureau of Mines, 1994. 38p.

LATORRACA, J. V. D. F.; RODRIGUES, N. D.; VIEIRA, M. C.; OHANA, C. C.; TEIXEIRA, J. G. Efeito da umidade da madeira na propagação de ondas mecânicas. Floresta e Ambiente, Rio de Janeiro, v. I8, n. 4, p. 45 I-459, 20 I I.

MARTINIS, R.; SOCCO, L. V.; SAMBUELLI, L.; NICOLOTTI, G.; SCHMITT, O.; BUCUR, V. Tomographie ultrasonore pour les arbres sur pied. Annals of Forest Science, Paris, v. 6I, n. 2, p. I57-I62, mar. 2004.

PADUELLI, M. C. Estudo da técnica de ondas de tensão como instrumento de avaliação interna de árvores urbanas. 2011. 159 p. Tese (Doutorado em Engenharia Urbana) - Universidade Federal de São Carlos, São Carlos.

PERLIN, L. P. Tomografia ultrassônica em concreto: desenvolvimento de ferramenta computacional. 20 I I. II4 p. Dissertação (Mestrado em Engenharia Civil) - Universidade Federal de Santa Catarina, Florianópolis.

PERLIN, L. P.; PINTO, R. C. A. Tomografia ultrassônica em concreto. Revista IBRACON de Estruturas e Materiais, São Paulo, v. 6, n. 2, p. 246-269, abr. 2013.

PRIETO, F.; LASAYGUES, P. Literature review of acoustic and ultrasonic tomography in standing trees. Trees, New York, v. 28, n. 6 , p. 1559-I567, 2014.

RADON, J. Über die Bestimmung von Funktionen durch ihre Integralwerte längs gewisser Mannigfaltigkeiten. Berichte der Sächsischen Akademie der Wissenschaft, Leipzig, v. 69 , p. 262-277, 1917.

RUGGIERO, M. A. G.; LOPES, V. L. R. Cálculo numérico: aspectos teóricos e computacionais. 2. ed. São Paulo: Pearson Makron Books, 1996. 406p.

SAMBUELLI, L.; SOCCO, L. V.; GODIO, A.; NICOLOTTI, G.; MARTINIS, R. Ultrasonic, electric and radar measurements for living trees assessment. Bollettino di Geofisica Teorica e Applicata, Torino, v. 44, n. 3, p. 253-279, set. 2003.

SECCO, C. B.; GONÇALVES, R.; CERRI, D. G. P.; VASQUES, É. C.; BATISTA, F. A. F. Behavior of ultrasonic waves in wood with presence of holes. Cerne, Larvas, v. 18, n. 3, p. 507-5 I4, mar. 2012.

WANG, X.; WIEDENBECK, J.; LIANG, S. Acoustic tomography for decay detection in black cherry trees. Wood and Fiber Science, Hanover, Pennsylvania, v. 4I, n. 2, p. I27I37, 2009. 